\title{
An Expanded Carrier Screening Tool Enhances Preconception Cystic Fibrosis Screening in Infertile Couples
}

\author{
Alexandra Higgins' ${ }^{1}$, Jason Flanagan ${ }^{2,3}$, Tiffany Von Wald ${ }^{2,3}$, Keith A. Hansen ${ }^{2,3}$ \\ ${ }^{1}$ The University of South Dakota Sanford School of Medicine, Sioux Falls, USA \\ ${ }^{2}$ Sanford Health Fertility and Reproductive Medicine, Sioux Falls, USA \\ ${ }^{3}$ Department of Ob/Gyn, The University of South Dakota Sanford School of Medicine, Sioux Falls, USA \\ Email: alexandra.higgins@usd.edu
}

Received 19 June 2015; accepted 21 July 2015; published 24 July 2015

Copyright (C) 2015 by authors and Scientific Research Publishing Inc.

This work is licensed under the Creative Commons Attribution International License (CC BY). http://creativecommons.org/licenses/by/4.0/

(c) (;) Open Access

\section{Abstract}

The American College of Obstetricians and Gynecologists recommends offering preconception and prenatal screening for cystic fibrosis (CF) while the American College of Medical Genetics also recommends screening for spinal muscular atrophy (SMA) to all couples. Both groups suggest specific screening if there is a family or personal history of a genetic disease or if the individual is from a high risk ethnic group. The purpose of this study was to determine whether availability of a more comprehensive, affordable genetic screening tool increased the number of infertility patients choosing to be screened for $\mathrm{CF}$ and other genetic diseases. This was a retrospective chart review of new infertility patients evaluated between May 2010 and May 2013. Sixteen hundred seventy-five new infertility couples were offered Counsyl ${ }^{\mathrm{TM}}$ expanded carrier screening. The carrier frequency for $\mathrm{CF}$ was $6.8 \%$ with $0 \%$ of the couples concordant heterozygotes. The carrier frequency for SMA was $2.51 \%$ with $0 \%$ of the couples concordant heterozygotes. With availability of the Counsyl ${ }^{\mathrm{TM}}$ screening test, the percentage of new infertility patients choosing to have preconception genetic screening increased from $2 \%$ to $8 \%$ in this population. The largest increase (17.5\% of new patients) in screening followed the reduction in out-of-pocket expense in May 2013. Infertility patients are in a unique position to investigate their family history, discuss appropriate preconception genetic screening, and, if discovered to be at high risk of a genetic illness, to review their reproductive options.

\section{Keywords}

Cystic Fibrosis, Preconception Screening, Spinal Muscular Atrophy, Infertility 


\section{Introduction}

Cystic fibrosis (CF) is a progressive, multisystem disease involving damage primarily to the pulmonary, pancreatic, and gastrointestinal systems. CF is caused by mutations in the cystic fibrosis transmembrane conductance regulator (CFTR) gene on chromosome 7. Over 1700 mutations for CF have been identified, although most cases of CF are caused by a small subset of these mutations. Over two-thirds of worldwide cases of CF are caused by a single mutation, $\Delta \mathrm{F} 508$. The carrier risk of $\mathrm{CF}$ in the non-Hispanic white population is approximately $4 \%$ and the disease incidence $0.04 \%$ making it the most common life threatening autosomal recessive condition in this population. The median life expectancy is 37 years old with respiratory failure being the primary cause of death [1].

In 2001, the American College of Obstetricians and Gynecologists (ACOG) recommended that all Caucasian couples be offered preconception CF screening [2]. The 2011 US Census Bureau data shows a continued increase in the number of people reporting mixed ancestry, particularly in the young, reproductive population [3]. As it is becoming increasingly difficult to assign a single ethnicity to individuals [4], ACOG now recommends that all patients be offered CF screening, although it is still most efficacious in the non-Hispanic white and Ashkenazi Jewish populations [5]. Additionally, the American College of Medical Genetics (ACMG) recommends screening all couples for spinal muscular atrophy [6], a severe neurodegenerative disease, while ACOG only recommends screening for those with a family history of SMA [7]. Both groups recommend screening for fragile $\mathrm{X}$ syndrome in patients with a family history of unexplained mental retardation, developmental delay or autism, or a personal or family history of ovarian insufficiency [8]. With the rapidly declining costs of DNA sequencing [9], the cost of screening for hundreds of genetic diseases including the formerly discussed is now less than the lifetime costs of treating an affected individual [10] [11].

In 2007, only $2 \%$ of patients evaluated for infertility at Sanford Health Fertility and Reproductive Medicine elected to have preconception screening for CF [12]. At that time, the cost of CF screening was approximately $\$ 500$ and it screened for 97 of the most common disease causing mutations. On May 20, 2010, Counsyl released an expanded carrier screening test. The expanded carrier screening tool screens for 106 genetic disorders including the 99 most common disease causing CF mutations. The maximum out of pocket cost to patients reduced from \$349 to \$99 in May 2012 and a combined Fragile X testing became available at the same cost (formerly a separate $\$ 600$ test). The aim of this study was to determine whether availability of a more comprehensive, affordable genetic screening tool increased the number of patients choosing to have preconception screening for cystic fibrosis.

\section{Materials and Methods}

This is an IRB approved retrospective chart review completed from June to July 2013 of new patients evaluated for primary or secondary infertility at Sanford Health Fertility and Reproductive Medicine between May 20, 2010 and May 20, 2013 (IRB 2013.095 approved 4/22/2013). A search of the electronic medical record was performed to identify all new infertility patients and a separate search was performed of the Counsyl database to identify all patients who submitted a sample for genetic testing. The two data sets were compared to ensure inclusion of all new patients and all identified records were reviewed and data abstracted. The abstracted data was organized into an electronic database using Excel and stored on a secure computer. Abstracted data included name, date of service, gender, ethnicity, partner testing, family history, IVF attempts, infertility status, and genetic screening results. Inclusion criteria for couples included evaluation for either primary or secondary infertility including both male and female factors. These factors included low or abnormal sperm counts, recurrent miscarriages, PCOS, ovarian dysfunction, uterine abnormalities, or female infertility of unknown origin. Couples seeking genetic counseling or referred for genetic counseling who did not have complaints of infertility were not included in this study. Occasionally, if an abnormality was found on screening, extended family members would seek genetic screening and these patients were also not included in this study.

Couples received a detailed family history and were offered the Counsyl expanded carrier screening test after genetic counseling. The percentage of these patients choosing to undergo preconception genetic screening was determined as well as the carrier frequencies of cystic fibrosis, spinal muscular atrophy, fragile X syndrome, and other genetic diseases. The mild diseases of Factor V Leiden deficiency, G6PD deficiency, HFE-associated hereditary hemochromatosis, pseudocholinesterase deficiency, and MTHFR deficiencies were excluded when determining the total percent of the population who were carriers of genetic diseases. 


\section{Results}

Between May 20, 2010 and May 20, 2013, 1669 new couples were evaluated for primary or secondary infertility and offered the Counsyl expanded carrier screening test. Of those offered, 134 couples (8\%) underwent screening of either one or both partners. Of the couples screened, $48.5 \%$ couples screened both partners, $44 \%$ screened only the female partner, and $7.5 \%$ screened only the male partner resulting in a total of 199 individuals being screened. One hundred ninety-three of the individuals (97\%) screened were non-Hispanic Caucasian, three were Asian, two were Middle Eastern, and one was Hispanic. Comparatively, ninety-four percent of the total cohort offered screening were non-Hispanic Caucasian with 1.9\% African-American, $1.8 \%$ Asian, and the remainder either American Indian, Hispanic, multiracial, or unknown. Only 3.3\% of couples evaluated before the price decrease to $\$ 99$ chose to be screened (37 of 1117 couples). After the price decrease to $\$ 99,17.5 \%$ of couples evaluated chose to be screened (97 of 552 couples) (Table 1 ).

Excluding mild diseases, thirty-one percent (62 of 199) of individuals were carriers for at least one serious genetic disease and 8\% (16/199) were carriers of at least two serious genetic diseases. No couples in this study population were found to be concordant heterozygotes of a serious genetic disease. Three of the couples choosing to screen reported a family history of CF. The carrier frequency for CF was $6.8 \%$ with $0 \%$ of the couples concordant heterozygotes. The carrier frequencies for SMA and Fragile X syndrome were $2.51 \%$ and $2.78 \%$, respectively. Additionally, $48.5 \%$ of the screened couples pursued in vitro fertilization after screening.

\section{Discussion}

Since the release of the Counsyl expanded carrier screening test in May 2010, the percentage of new infertility patients at this Midwest infertility clinic choosing to undergo preconception genetic screening increased from $2 \%$ to $8 \%$. It appears that the price reduction of the Counsyl test played a key role in a patient's decision to screen. Before the price decrease only $3.3 \%$ of couples were screened. After the price decrease $17.5 \%$ of patients were screened (Table 1). One hundred dollars may represent a threshold price that patients are willing to pay for genetic screening.

The carrier frequency for CF was found to be $6.8 \%$ in this study as compared with the anticipated $4 \%$ carrier risk before testing. The carrier frequency for SMA $2.51 \%$, close to the expected carrier risk and the fragile $\mathrm{X}$ syndrome carrier frequency was $2.78 \%$. These carrier frequencies are compared to those found in a large study by Lazarin et al. of over 23,000 patients in 2013 (Table 2) [13]. Excluding mild diseases, twenty-four percent of individuals in the Lazarin et al. study were found to be carriers of at least one genetic disease while $5.2 \%$ were carriers of at least two serious genetic diseases. The results of this smaller study were comparable, with $31 \%$ of individuals being carriers for at least one serious genetic disease and $8 \%$ carriers of at least two serious genetic diseases. No couples in this study were found to be concordant heterozygotes of a serious genetic disease; however, that was not surprising given this small study population.

Rapidly declining cost of DNA sequencing is making preconception screening much more cost-effective. Additionally, increasing difficulty in assigning a single ethnicity to individuals is causing pan-ethnic screening to appear to be a more reliable way of screening individuals and finding these rare and life-threatening genetic diseases. Patients seeking evaluation for infertility are in a unique position to discuss preconception genetic screening. Couples found to both be heterozygous for a cystic fibrosis mutation can be offered information about donor sperm or eggs, donor embryos, IVF with preimplantation genetic diagnosis, and prenatal diagnosis. More couples may choose to pursue preconception genetic screening for cystic fibrosis and other serious genetic disorders with continued improvement, expansion, and price reduction of genetic carrier screening tools.

Table 1. Preconception genetic screening compared to cost of test. Percentage of couples evaluated for primary or secondary infertility choosing to pursue preconception genetic screening during three different time periods.

\begin{tabular}{cccc}
\hline Time Period & Cost of Counsyl test & \# of couples evaluated & \% of couples screened \\
\hline $5 / 20 / 2010-4 / 30 / 2012$ & $\$ 349$ & 1117 & $3.3 \%$ \\
$5 / 1 / 2012-5 / 20 / 2013$ & $\$ 99$ & 552 & $17.6 \%$ \\
Combined time period 5/20/2010-5/20/2013 & $\$ 99-\$ 349$ & Total 1669 & $8 \%$ \\
\hline
\end{tabular}


Table 2. Carrier frequencies and screening recommendations for cystic fibrosis, spinal muscular atrophy, and fragile X syndrome.

\begin{tabular}{cccc}
\hline Disease & $\begin{array}{c}\text { Counsyl Carrier } \\
\text { Frequency (\%) } \\
\text { NW European }\end{array}$ & $\begin{array}{c}\text { Carrier Frequency } \\
\text { (\%, infertility patients) } \\
\mathbf{N}=\mathbf{1 9 9}\end{array}$ & $\begin{array}{c}\text { ACOG } \\
\text { ACMG } \\
\text { Cystic Fibrosis }\end{array}$ \\
recommendations \\
\hline Spinal Muscular Atrophy & 2.57 & 6.79 & Screen all couples \\
Fragile X syndrome & 0.38 & 2.51 & Screen with indication \\
\hline
\end{tabular}

This table compares the carrier frequency of cystic fibrosis, fragile X syndrome, and spinal muscular found by Counsyl compared to our smaller population of infertility patients. ACOG and ACMG screening recommendations are also shown. Both ACOG and ACMG recommend preconception screening for cystic fibrosis for all patients, regardless of ethnicity. ACMG recommends SMA screening for all patients while ACOG recommends SMA screening for those patients with a family history of SMA or patients who request screening. Both ACOG and ACMG recommend Fragile X screening for patients with a family history of unexplained mental retardation, developmental delay or autism, or a personal or family history of ovarian insufficiency. Additionally, ACOG recommends screening all couples of Ashkenazi Jewish descent for Tay-Sachs, familial dysautonomia, and Canavan Disease. Genetic screening may be appropriate for patients with a family history of any genetic disease.

\section{Conclusion}

The number of infertility patients choosing to undergo preconception genetic screening increased significantly after the release of an expanded carrier screening tool. The percent of patients screening rose from $3.3 \%$ to $17.5 \%$ after the price reduction to ninety-nine dollars. Over $31 \%$ were carriers of at least one life-threatening disease and $8 \%$ were carriers of two of more diseases. The carrier frequencies of cystic fibrosis and fragile $\mathrm{X}$ syndrome were higher than expected $(6.8 \%$ and $2.78 \%$ respectively) and the carrier frequency of SMA was close to expected (2.51\%). None of the couples were found to be concordant heterozygotes.

\section{References}

[1] Moskowitz, S.M., Chmiel, J.F., Sternen, D.L., Cheng, E., Gibson, R.L., Marshall, S.G., et al. (2008) Clinical Practice and Genetic Counseling for Cystic Fibrosis and CFTR-Related Disorders. Genetics in Medicine, 10, 851-868. http://dx.doi.org/10.1097/GIM.0b013e31818e55a2

[2] Fries, M.H., Bashford, M. and Nunes, M. (2005) Implementing Prenatal Screening for Cystic Fibrosis in Routine Obstetric Practice. American Journal of Obstetrics \& Gynecology, 192, 527-534.

http://dx.doi.org/10.1016/j.ajog.2004.07.028

[3] US Census Bureau (2012) The Statistical Abstract of the United States: 2012. 131st Edition.

[4] Ross, L.F. (2012) A Re-Examination of the Use of Ethnicity in Prenatal Carrier Testing. American Journal of Medical Genetics Part A, 158a, 19-23. httsp://dx.doi.org/10.1002/ajmg.a.34361

[5] (2011) ACOG Committee Opinion No. 486: Update on Carrier Screening for Cystic Fibrosis. Obstetrics \& Gynecology, 117, 1028-1031. http://dx.doi.org/10.1097/AOG.0b013e31821922c2

[6] Prior, T.W. (2008) Carrier Screening for Spinal Muscular Atrophy. Genetics in Medicine, 10, 840-842. http://dx.doi.org/10.1097/GIM.0b013e318188d069

[7] (2009) ACOG Committee Opinion No. 432: Spinal Muscular Atrophy. Obstetrics \& Gynecology, 113, 1194-1196. http://dx.doi.org/10.1097/AOG.0b013e3181a6d03a

[8] (2010) ACOG Committee Opinion No. 469: Carrier Screening for Fragile X Syndrome. Obstetrics \& Gynecology, 116, 1008-1010. http://dx.doi.org/10.1097/AOG.0b013e3181fae884

[9] Wetterstrand, K.A. (2014) DNA Sequencing Costs: Data from the NHGRI Genome Sequencing Program (GSP). www.genome.gov/sequencingcosts

[10] Wei, S.Q.M. and Monaghan, K.G. (2007) Is Cystic Fibrosis Carrier Screening Cost Effective? Community Genetics, 10, 7. http://dx.doi.org/10.1159/000099088

[11] Srinivasan, B.S., Evans, E.A., Flannick, J., Patterson, A.S., Chang, C.C., Pham, T., et al. (2010) A Universal Carrier Test for the Long Tail of Mendelian Disease. Reproductive BioMedicine Online, 21, 537-551. http://dx.doi.org/10.1016/j.rbmo.2010.05.012

[12] Slostad, J., Stein, Q.P., Flanagan, J.D. and Hansen, K.A. (2007) Screening for Mutations in the Cystic Fibrosis Transmembrane Regulator Gene in an Infertility Clinic. Fertility and Sterility, 88, 1687-1688. 
http://dx.doi.org/10.1016/j.fertnstert.2007.01.065

[13] Lazarin, G.A., Haque, I.S., Nazareth, S., Iori, K., Patterson, A.S., Jacobson, J.L., et al. (2013) An Empirical Estimate of Carrier Frequencies for 400+ Causal Mendelian Variants: Results from an Ethnically Diverse Clinical Sample of 23,453 Individuals. Genetics in Medicine, 15, 178-186. 